\title{
Isometric group actions on Hilbert spaces: growth of cocycles
}

\author{
Yves de Cornulier, Romain Tessera, Alain Valette
}

October 8, 2018

\begin{abstract}
We study growth of 1-cocycles of locally compact groups, with values in unitary representations. Discussing the existence of 1-cocycles with linear growth, we obtain the following alternative for a class of amenable groups $G$ containing polycyclic groups and connected amenable Lie groups: either $G$ has no quasi-isometric embedding into a Hilbert space, or $G$ admits a proper cocompact action on some Euclidean space.

On the other hand, noting that almost coboundaries (i.e. 1-cocycles approximable by bounded 1-cocycles) have sublinear growth, we discuss the converse, which turns out to hold for amenable groups with "controlled" Følner sequences; for general amenable groups we prove the weaker result that 1-cocycles with sufficiently small growth are almost coboundaries. Besides, we show that there exist, on a-T-menable groups, proper cocycles with arbitrary small growth.
\end{abstract}

Mathematics Subject Classification: Primary 22D10; Secondary 43A07, 43A35, 20F69.

Key words and Phrases: Haagerup property, a-T-menability, amenability, growth of cocycles, Hilbert distances, geometric group theory, Bernstein functions.

Notation. Let $G$ be a locally compact group, and $f, g: G \rightarrow \mathbf{R}_{+}$. We write $f \preceq g$ if there exists $M>0$ and a compact subset $K \subset G$ such that $f \leq M g$ outside $K$. We write $f \sim g$ if $f \preceq g \preceq f$. We write $f \prec g$ if, for every $\varepsilon>0$, there exists a compact subset $K \subset G$ such that $f \leq \varepsilon g$ outside $K$.

\section{Introduction}

The study of affine isometric actions on Hilbert spaces has proven to be a fundamental tool in geometric group theory. Let $G$ be a locally compact group, and 
$\alpha$ an affine isometric action on an affine Hilbert space $\mathcal{H}$ (real or complex). The function $b: G \rightarrow \mathcal{H}$ defined by $b(g)=\alpha(g)(0)$ is called a 1-cocycle (see Section 2 for details), and we call the function $g \mapsto\|b(g)\|$ a Hilbert length function on $G$. We focus on the asymptotic behaviour of Hilbert length functions on a given group $G$. A general question is the following: how is it related to the structure of $G$ ?

For instance, if $G$ is $\sigma$-compact, $G$ has the celebrated Kazhdan's Property (T) if and only if every Hilbert length function is bounded (see [HV]). This is known to have strong group-theoretic consequences on $G$ : for instance, this implies that $G$ is compactly generated and has compact abelianization (see [BHV, Chap. 2] for a direct proof).

In this paper, we rather deal with groups which are far from having Kazhdan's Property (T): a locally compact group $G$ is called a-T-menable if it has a proper Hilbert length function. The class of a-T-menable locally compact groups contains (see [CCJJV]) amenable groups, Coxeter groups, isometry groups of locally finite trees, isometry groups of real and complex hyperbolic spaces and all their closed subgroups, such as free and surface groups. We show in $\$ 3.4$ that, for a-T-menable locally compact groups (for instance, $\mathbf{Z}$ ), there exist proper Hilbert length functions of arbitrary slow growth.

The study of Hilbert length functions with non-slow growth is more delicate. An easy but useful observation is that, for a given compactly generated, locally compact group, any Hilbert length function $L$ is linearly bounded, i.e. $L(g) \preceq$ $|g|_{S}$, where $|\cdot|_{S}$ denotes the word length with respect to some compact generating subset.

We discuss, in Section 3, Hilbert length functions with sublinear growth. These include those Hilbert length functions whose corresponding 1-cocycle (see Section 2) is an almost coboundary, i.e. can be approximated, uniformly on compact subsets, by bounded 1-cocycles. We discuss the converse.

Denote by $(\mathcal{L})$ the class of groups including:

- polycyclic groups and connected amenable Lie groups,

- semidirect products $\mathbf{Z}\left[\frac{1}{m n}\right] \rtimes_{\frac{m}{n}} \mathbf{Z}$, with $m, n$ co-prime integers with $|m n| \geq 2$ (if $n=1$ this is the Baumslag-Solitar group $B S(1, m)$ ); semidirect products $\left(\mathbf{R} \oplus \bigoplus_{p \in S} \mathbf{Q}_{p}\right) \rtimes_{\frac{m}{n}} \mathbf{Z}$ or $\left(\bigoplus_{p \in S} \mathbf{Q}_{p}\right) \rtimes_{\frac{m}{n}} \mathbf{Z}$, with $m, n$ co-prime integers, and $S$ a finite set of prime numbers dividing $m n$.

- wreath products $F \nmid \mathbf{Z}$ for $F$ a finite group.

Theorem 1.1 (see Corollary 3.7, Propositions 3.5 and 3.9). 
(1) If $G$ is a compactly generated, locally compact amenable group, then every 1-cocycle with sufficiently slow growth is an almost boundary.

(2) For groups in the class $(\mathcal{L})$, every sublinear 1-cocycle is an almost coboundary.

(3) If $\Gamma$ is a finitely generated, discrete, non-amenable subgroup in $\mathrm{SO}(n, 1)$ or $\mathrm{SU}(n, 1)$ for some $n \geq 2$, then $\Gamma$ admits a 1-cocycle with sublinear growth (actually $\preceq|g|^{1 / 2}$ ) which is not an almost coboundary.

In 93.5 , we show that there exist, on $\mathbf{R}^{n}$ or $\mathbf{Z}^{n}$, Hilbert length functions with arbitrary large sublinear growth, showing that, in a certain sense, there is no gap between Hilbert length functions of linear and of sublinear growth.

In Section 4, we discuss the existence of a Hilbert length function on $G$ with linear growth. Such a function exists when $G=\mathbf{Z}^{n}$. We conjecture that the converse is essentially true.

Conjecture 1. Let $G$ be a locally compact, compactly generated group having a Hilbert length function with linear growth. Then $G$ has a proper, cocompact action on a Euclidean space. In particular, if $G$ is discrete, then it must be virtually abelian.

Our first result towards Conjecture 1 is a generalization of a result by Guentner and Kaminker [GK, §5] to the non-discrete case.

Theorem 1.2 (see Theorem4.1). Let $G$ be a locally compact, compactly generated group. If $G$ admits a Hilbert length function with growth $\succ|g|^{1 / 2}$ (in particular, if it admits a Hilbert length function with linear growth), then $G$ is amenable.

We actually provide a new, simpler proof, while it is not clear how to generalize the proof in GK to the non-discrete case 1 .

To prove that locally compact groups in the class $(\mathcal{L})$ satisfy Conjecture 1 , we use Shalom's Property $H_{F D}$ : a locally compact group has Property $H_{F D}$ if any unitary representation with nontrivial reduced cohomology has a finitedimensional nonzero subrepresentation. All groups in the class $(\mathcal{L})$ are known to satisfy Property $H_{F D}$. We prove

Theorem 1.3 (see Theorem 4.3). Locally compact, compactly generated groups with Property $H_{F D}$ satisfy Conjecture 1 .

\footnotetext{
${ }^{1}$ The truncation argument in $[\mathrm{GK}]$ does not seem to extend to non-discrete groups.
} 
We next consider uniform embeddings into Hilbert spaces. There is a nice trick, for which we are indebted to Gromov, allowing to construct, if the group is amenable, a 1-cocycle with the same growth behaviour as the initial embedding. See Proposition 4.4 for a precise statement. Thus we get:

Theorem 1.4. If $G$ is any locally compact, compactly generated, amenable group with Property $H_{F D}$ (e.g. in the class $(\mathcal{L})$ ), then

- either $G$ does not admit any quasi-isometric embedding into a Hilbert space,

- or $G$ acts properly cocompactly on some Euclidean space (i.e. a finitedimensional real Hilbert space).

The reader interested in the proof of Theorem 1.4 can skip Section 3, except the elementary Proposition 3.1. Let us observe that the proof of Theorem 1.4 does not appeal to asymptotic cones. It contains, as a particular case, the fact that a simply connected nilpotent non-abelian Lie group has no quasi-isometric embedding into a Hilbert space, a result due to S. Pauls [Pau]. Moreover, Theorem 1.4 provides new proofs of two known results (see $\$ 4.3$ for proofs):

Corollary 1.5 (Quasi-isometric rigidity of $\mathbf{Z}^{n}$ ). If a finitely generated group is quasi-isometric to $\mathbf{Z}^{n}$, then it has a finite index subgroup isomorphic to $\mathbf{Z}^{n}$.

This latter result has recently been proved by Shalom [Sha04, who first establishes the invariance of Property $H_{F D}$ by quasi-isometries. We also make use of this crucial fact, although the use of Proposition 4.4 allows us to conclude in a different way.

Corollary 1.6 (Bourgain [Bou]). For $r \geq 3$, the regular tree of degree $r$ does not embed quasi-isometrically into a Hilbert space.

A locally compact, compactly generated group is either non-amenable or nonunimodular if and only if it is quasi-isometric to a graph with positive Cheeger constant (see [Tes2, Corollary 7.12]). In Corollary 1.6 of [BeSc, Benjamini and Schramm use Bourgain's result above, to prove that a graph with positive Cheeger constant cannot be quasi-isometrically embedded into a Hilbert space. As a consequence: if a compactly generated, locally compact group $G$ admits a quasiisometric embedding into Hilbert space, then $G$ is amenable and unimodular.

By Proposition 4.4, the existence of a quasi-isometric embedding into a Hilbert space implies, for an amenable group, the existence of a Hilbert length function with linear growth. So Conjecture 1 is equivalent to the following statement, apparently more general: 
Conjecture 1'A locally compact, compactly generated group $G$ admitting a quasi-isometric embedding into a Hilbert space has a proper, cocompact action on a Euclidean space. In particular, if $G$ is discrete, then it must be virtually abelian.

We conclude this introduction with a remark about compression. The following definition is due to E. Guentner and J. Kaminker [GK]. Let $G$ be a compactly generated group, endowed with its word length $|\cdot|_{S}$.

Definition 1.7. The equivariant Hilbert space compression of $G$ is defined as:

$$
B(G)=\sup \left\{\alpha \geq 0, \exists \text { unitary representation } \pi \quad \exists b \in Z^{1}(G, \pi),\|b(g)\| \succeq|g|_{S}^{\alpha}\right\} \text {. }
$$

It is clear that $0 \leq B(G) \leq 1$, and if $G$ admits a linear 1-cocycle, then $B(G)=1$. The converse in not true: it is shown in [Tes1] that $B(G)=1$ for groups of the class $(\mathcal{L})$ whereas we have shown above (Theorem 1.4) that these groups do not admit linear cocycles unless they act properly on a Euclidean space.

Another immediate observation is that if $B(G)>0$, then $G$ is a-T-menable. We know nothing about the converse: actually we know no example of an a-Tmenable group with $B<1 / 2$; at the other extreme, we do not know if solvable groups always satisfy $B>0$.

It follows from Proposition 4.4 that, for amenable groups $G$, the number $B(G)$ is a quasi-isometry invariant. This probably does not hold for non-amenable groups, but we do not know any counterexample. More precisely

- It is not known if being a-T-menable is a quasi-isometry invariant.

- It is not known if there exists a non-amenable, a-T-menable, compactly generated, locally compact group $G$ with $B(G) \neq 1 / 2$.

Finally, let us mention that it can be interesting to study the growth of 1cocycles when we restrict to certain special classes of unitary representations. In the case of the regular representation, some results can be found in [Tes1], related to isoperimetric properties of the group.

Acknowledgments. We are indebted to Misha Gromov for a decisive remark. We also thank Emmanuel Breuillard, Pierre de la Harpe, and Urs Lang for useful remarks and corrections. 


\section{Preliminaries}

\subsection{Growth of 1-cocycles}

Let $G$ be a locally compact group, and $\pi$ a unitary or orthogonal representation (always assumed continuous) on a Hilbert space $\mathcal{H}=\mathcal{H}_{\pi}$. The space $Z^{1}(G, \pi)$ is defined as the set of continuous functions $b: G \rightarrow \mathcal{H}$ satisfying, for all $g, h \in G$, the 1-cocycle condition $b(g h)=\pi(g) b(h)+b(g)$. Observe that, given a continuous function $b: G \rightarrow \mathcal{H}$, the condition $b \in Z^{1}(G, \pi)$ is equivalent to saying that $G$ acts by affine transformations on $\mathcal{H}$ by $\alpha(g) v=\pi(g) v+b(g)$. The space $Z^{1}(G, \pi)$ is endowed with the topology of uniform convergence on compact subsets.

The subspace of coboundaries $B^{1}(G, \pi)$ is the subspace (not necessarily closed) of $Z^{1}(G, \pi)$ consisting of functions of the form $g \mapsto v-\pi(g) v$ for some $v \in \mathcal{H}$. It is well-known [HV, §4.a] that $b \in B^{1}(G, \pi)$ if and only if $b$ is bounded on $G$.

The subspace of almost coboundaries $\overline{B^{1}(G, \pi)}$ is the closure of $B^{1}(G, \pi)$. A 1-cocycle $b$ is an almost coboundary if and only if the corresponding affine action almost has fixed points, i.e. for every compact subset $K \subset G$ and $\varepsilon>0$, there exists $v$ such that $\sup _{g \in K}\|\alpha(g) v-v\| \leq \varepsilon$ (see [BHV, §3.1]). When $G$ is generated by a symmetric compact subset $S$, it suffices to check this condition for $K=S$, and a sequence of almost fixed points is defined as a sequence $\left(v_{n}\right)$ such that $\sup _{g \in S}\left\|\alpha(g) v_{n}-v_{n}\right\| \rightarrow 0$.

The first cohomology space of $\pi$ is defined as the quotient space $H^{1}(G, \pi)=$ $Z^{1}(G, \pi) / B^{1}(G, \pi)$, and the first reduced cohomology space of $\pi$ is defined as $\overline{H^{1}}(G, \pi)=Z^{1}(G, \pi) / \overline{B^{1}(G, \pi)}$.

Now suppose that $G$ is a locally compact, compactly generated group. For $g \in G$, denote by $|g|_{S}$ the word length of $g$ with respect to an open, relatively compact generating set $S \subset G$.

Let $b \in Z^{1}(G, \pi)$ be a 1 -cocycle with respect to a unitary representation $\pi$ of $G$. We study the growth of $\|b(g)\|$ as a function of $g$.

Definition 2.1. The compression of the 1-cocycle $b$ is the function

$$
\rho: \mathbf{R}_{+} \rightarrow \mathbf{R}_{+} \cup\{\infty\}: x \mapsto \rho(x)=\inf \left\{\|b(g)\|: g \in G,|g|_{S} \geq x\right\} .
$$

Remark 2.2. A related notion is the distortion function, defined in [Far] in the context of an embedding between finitely generated groups. The distortion function of the 1-cocycle $b$ is defined as the function $\mathbf{R}_{+} \rightarrow \mathbf{R}_{+} \cup\{\infty\}$ by $f(x)=$ $\sup \left\{|g|_{S}:\|b(g)\| \leq x\right\}$. The reader can check that, except in trivial cases 2 , the

\footnotetext{
${ }^{2}$ Trivial cases are: when $G$ is compact, so that $\rho$ is eventually equal to $\infty$ and $f$ is eventually equal to a finite constant, and when $b$ is not proper, so that $\rho$ is bounded, and $f$ is eventually equal to $\infty$.
} 
compression $\rho$ and the distortion $f$ are essentially reciprocal to each other.

Recall that a length function on a group $\Gamma$ is a function $L: \Gamma \rightarrow \mathbf{R}_{+}$satisfying $L(1)=0$ and, for all $g, h \in \Gamma, L\left(g^{-1}\right)=L(g)$ and $L(g h) \leq L(g)+L(h)$, so that $d(g, h)=L\left(g^{-1} h\right)$ is a left-invariant pseudo-distance ("écart") on $\Gamma$.

It is immediate from the 1-cocycle relation that the function $g \mapsto\|b(g)\|$ is a length function on the group $G$. In particular, if $G$ is locally compact, compactly generated, then it is dominated by the word length. We thus obtain the following obvious bound:

Proposition 2.3. For $b \in Z^{1}(G, \pi)$, we have $\|b(g)\| \preceq|g|_{S}$.

Define

$$
\begin{gathered}
\operatorname{lin}(G, \pi)=\left\{b \in Z^{1}(G, \pi),\|b(g)\| \succeq|g|_{S}\right\} \\
\operatorname{sublin}(G, \pi)=\left\{b \in Z^{1}(G, \pi),\|b(g)\| \prec|g|_{S}\right\},
\end{gathered}
$$

namely, the set of cocycles with linear (resp. sublinear) growth. Here are immediate observations:

- $\operatorname{sublin}(G, \pi)$ is a linear subspace of $Z^{1}(G, \pi)$.

- $B^{1}(G, \pi) \subset \operatorname{sublin}(G, \pi) \subset Z^{1}(G, \pi) \backslash \operatorname{lin}(G, \pi)$.

- If $G=\mathbf{Z}$ or $\mathbf{R}$, then it is easy to check that $Z^{1}(G, \pi)=\operatorname{lin}(G, \pi) \cup$ $\operatorname{sublin}(G, \pi)$ (this follows either from Corollary 3.7 below, or from a direct computation 3 ). On the other hand, this does not generalize to arbitrary $G$. Indeed, take any nontrivial action of $\mathbf{Z}^{2}$ by translations on $\mathbf{R}$ : then the associated cocycle is neither linear nor sublinear.

\subsection{Conditionally negative definite functions and Bern- stein functions}

A conditionally negative definite function on a group $G$ is a function $\psi: G \rightarrow \mathbf{R}_{+}$ such that $\psi^{1 / 2}$ is a Hilbert length function. Equivalently [HV, 5.b], $\psi(1)=0$, $\psi(g)=\psi\left(g^{-1}\right)$ for all $g$, and, for all $\lambda_{1}, \ldots, \lambda_{n} \in \mathbf{R}$ such that $\sum_{i=1}^{n} \lambda_{i}=0$ and for all $g_{1}, \ldots, g_{n} \in G$, we have $\sum_{i, j=1}^{n} \lambda_{i} \lambda_{j} \psi\left(g_{i}^{-1} g_{j}\right) \leq 0$. Continuous conditionally

\footnotetext{
${ }^{3}$ Here is the argument for $\mathbf{Z}$. Fix $b \in Z^{1}(\mathbf{Z}, \pi)$. Write $U=\pi(1)$ and $\pi=\pi_{0} \oplus \pi_{1}$, where $\pi_{0}=\operatorname{Ker}(U-1)$ denotes the invariant vectors, and decompose $b$ as $b_{0}+b_{1}$. Clearly, $b_{0}$ is either zero or has linear growth. On the other hand, $b_{1}$ has sublinear growth: indeed, as $b_{1}(1)$ is orthogonal to $\operatorname{Ker}(U-1)$, it belongs to $\overline{\operatorname{Im}(U-1)}$, so that $b_{1} \in \overline{B^{1}\left(\mathbf{Z}, \pi_{1}\right)}$. Hence $b_{1} \in \operatorname{sublin}(\mathbf{Z}, \pi)$ by Corollary 3.3 below.
} 
negative definite functions on a locally compact group $G$ form a convex cone, closed under the topology of uniform convergence on compact subsets.

A continuous function $F: \mathbf{R}_{+} \rightarrow \mathbf{R}_{+}$is a Bernstein function if there exists a positive measure $\mu$ on Borel subsets of $\mathbf{R}_{+}^{*}$ such that $\mu([\varepsilon, \infty[)<\infty$ for all $\varepsilon>0$, $\int_{0}^{1} x d \mu(x)<\infty$, and such that, for some $a \geq 0$,

$$
\forall t>0, \quad F(t)=a t+\int_{0}^{+\infty}\left(1-e^{-t x}\right) d \mu(x)
$$

Note that such a function is real analytic on $\mathbf{R}_{+}^{*}$. We note for reference the following well-known result due to of Bochner and Schoenberg [Sch, Theorem 8]:

Lemma 2.4. Let $\psi$ be a conditionally negative definite function on $G$, and let $F$ be a Bernstein function. Then $F \circ \psi$ is conditionally negative definite on $G$.

Examples of Bernstein functions are $x \mapsto x^{a}$ for $0<a \leq 1$, and $x \mapsto \log (x+1)$. For more on Bernstein functions, see for instance $[\mathrm{BF}]$.

\section{Cocycles with sublinear growth}

\subsection{Almost coboundaries are sublinear}

Proposition 3.1. Let $G$ be a locally compact, compactly generated group. In $Z^{1}(G, \pi)$, endowed with topology of uniform convergence on compact subsets,

1) $\operatorname{sublin}(G, \pi)$ is a closed subspace;

2) $\operatorname{lin}(G, \pi)$ is an open subset.

Proof: Fix a symmetric open, relatively compact generating subset $S \subset G$. Let $b$ be the limit of a net $\left(b_{i}\right)_{i \in I}$ in $Z^{1}(G, \pi)$. Write $b_{i}^{\prime}=b-b_{i}$, and fix $\varepsilon>0$. For $i$ large enough (say, $\left.i \geq i_{0}\right), \sup _{s \in S}\left\|b_{i}^{\prime}(s)\right\| \leq \varepsilon / 2$. Since $g \mapsto\left\|b_{i}^{\prime}(g)\right\|$ is a length function, this implies that for every $g \in G$ and $i \geq i_{0},\left\|b_{i}^{\prime}(g)\right\| \leq \varepsilon|g|_{S} / 2$, i.e. $\left\|b_{i}^{\prime}(g)\right\| /|g|_{S} \leq \varepsilon / 2$.

1) Suppose that all $b_{i}$ 's belong to $\operatorname{sublin}(G, \pi)$. Fix $i \geq i_{0}$. Then $\left\|b_{i}(g)\right\| /|g|_{S} \leq$ $\varepsilon / 2$ for $g$ large enough (say, $g \notin K$ compact). So $\|b(g)\| /|g|_{S} \leq \varepsilon$ for $g \notin K$. This shows that $b \in \operatorname{sublin}(\Gamma, \pi)$, so we are done.

2) Suppose that $b \in \operatorname{lin}(G, \pi)$. Then, if $\varepsilon$ has been chosen sufficiently small, $\|b(g)\| /\|g\| \geq \varepsilon$ for large $g$ (say, $g \notin K$ compact). Hence, $\left\|b_{i}(g)\right\| /|g|_{S} \geq$ $\left(\|b(g)\|-\left\|b_{i}^{\prime}(g)\right\|\right) /|g|_{S} \geq \varepsilon / 2$ for $i \geq i_{0}, g \notin K$, showing that $b_{i} \in \operatorname{lin}(G, \pi)$ for $i \geq i_{0}$. 
Remark 3.2. In the previous result, it is essential that we fix the unitary representation $\pi$. Indeed, it is easy to show that, on every group $G$, every Hilbert length function (e.g. of linear growth) can be approximated, uniformly on compact subsets, by bounded Hilbert length functions.

Corollary 3.3. If $b \in \overline{B^{1}}(G, \pi)$, then $\|b(g)\| \prec|g|_{S}$.

Proof: $B^{1}(G, \pi) \subset \operatorname{sublin}(G, \pi)$, so that

$$
\overline{B^{1}(G, \pi)} \subset \overline{\operatorname{sublin}(G, \pi)}=\operatorname{sublin}(G, \pi)
$$

by Proposition 3.1 .

\subsection{Groups with controlled Følner sequences}

In this section, we prove that the converse to Corollary 3.3 is true for unimodular groups in the class $(\mathcal{L})$ : that is, a cocycle has sublinear growth if and only if it is an almost coboundary.

Let $G$ be a compactly generated, locally compact group with Haar measure $\mu$, and let $S$ be a compact generating subset. Let $\left(F_{n}\right)$ be a sequence of measurable, bounded subsets of nonzero measure. Set

$$
\varepsilon_{n}=\frac{\sup _{s \in S} \mu\left(s F_{n} \triangle F_{n}\right)}{\mu\left(F_{n}\right)} .
$$

Consider an isometric affine action $\alpha$ of $G$ on a Hilbert space, and let $b$ be the corresponding 1-cocycle. Set

$$
v_{n}=\frac{1}{\mu\left(F_{n}\right)} \int_{F_{n}} b(g) d \mu(g) .
$$

This is well-defined.

Lemma 3.4. Suppose that $\sup _{g \in F_{n}}\|b(g)\| \prec 1 / \varepsilon_{n}$. Then $\left(v_{n}\right)$ is a sequence of almost fixed points for the affine action $\alpha$ associated with $b$.

Proof: For $s \in S$, we have

$$
\alpha(s) v_{n}-v_{n}=\frac{1}{\mu\left(F_{n}\right)} \int_{F_{n}}(b(s g)-b(g)) d \mu(g) .
$$

Thus

$$
\left\|\alpha(s) v_{n}-v_{n}\right\| \leq \frac{2}{\mu\left(F_{n}\right)} \int_{s F_{n} \triangle F_{n}}\|b(g)\| d \mu(g) \leq 2 \varepsilon_{n} \sup _{g \in F_{n}}\|b(g)\| .
$$


Recall (see [BHV], Appendix G]) that " $G$ is amenable" exactly means that we can choose $\left(F_{n}\right)$ so that $\varepsilon_{n} \rightarrow 0$, and $\left(F_{n}\right)$ is then called a Følner sequence. In this case, we obtain, as a consequence of Lemma 3.4, that a 1-cocycle of sufficiently slow growth (depending on the behaviour of the Følner sequence, i.e. on the asymptotic behaviour of $\varepsilon_{n}$ and the diameter of $\left(F_{n}\right)$ ) must be an almost coboundary. We record this as:

Proposition 3.5. Let $G$ be a compactly generated, locally compact amenable group. Then there exists a function $u: G \rightarrow \mathbf{R}_{+} \cup\{\infty\}$ satisfying

- $\lim _{g \rightarrow \infty} u(g)=\infty$;

- for every 1-cocycle $b$ of $G,\|b(g)\| \prec u(g)$ implies that $b$ is an almost coboundary.

In other words, if two 1-cocycles are sufficiently close, then they coincide in reduced 1-cohomology.

Explicitly, the function $u$ can be defined as follows

$$
u(g)=\frac{1}{\max \left\{\varepsilon_{n} \mid n \in \mathbf{N} \text { s.t. } g \in F_{n}\right\}},
$$

where we set $u(g)=\infty$ if $g \notin \bigcup F_{n}$.

To obtain stronger statements we introduce a more restrictive notion of Følner sets.

Definition 3.6. We say that the Følner sequence $\left(F_{n}\right)$ of the amenable, compactly generated locally compact group $G$ is controlled if there exists a constant $c>0$ such that, for all $n$,

$$
F_{n} \subset B\left(1, c / \varepsilon_{n}\right)
$$

In [Tes1], it is proved that a unimodular group in the class $(\mathcal{L})$ admits a controlled Følner sequence.

Corollary 3.7. Let $G$ be a compactly generated, locally compact amenable group admitting a controlled Følner sequence $\left(F_{n}\right)$, and keep the notation as above. Then the following statements are equivalent:

(1) $b \in \overline{B^{1}}(\Gamma, \pi)$

(2) $b \in \operatorname{sublin}(\Gamma, \pi)$

(3) The sequence $\left(v_{n}\right)$ is a sequence of almost fixed points for $\alpha$. 
Proof: $(3) \Rightarrow(1)$ is immediate, while $(1) \Rightarrow(2)$ follows from Corollary 3.3. The remaining implication is $(2) \Rightarrow(3)$ : suppose that $b$ is sublinear. Write

$$
f(r)=\sup _{|g| \leq r}\|b(g)\|
$$

where $f(r) \prec r$. Then

$$
\sup _{g \in F_{n}}\|b(g)\| \leq \sup _{|g| \leq c / \varepsilon_{n}}\|b(g)\|=f\left(c / \varepsilon_{n}\right) \prec 1 / \varepsilon_{n}
$$

so that we can apply Lemma 3.4 to obtain that $\left(v_{n}\right)$ is a sequence of almost invariant vectors.

We use this to prove a conjecture of Shalom [Sha04, Section 6]. Recall that a representation of a group $\Gamma$ is said to be finite if it factors through a finite group.

Proposition 3.8. Let $\pi$ be a unitary representation of a finitely generated, virtually nilpotent group $\Gamma$ and let $S$ be a finite generating subset of $\Gamma$. Suppose that $\pi$ has no finite subrepresentation. For every cocycle $b \in Z^{1}(\Gamma, \pi)$, define:

$$
v_{n}=\frac{1}{\left|S^{n}\right|} \sum_{g \in S^{n}} b(g) .
$$

Then there exists a subsequence $\left(v_{n_{i}}\right)$ which is a sequence of almost fixed points for the affine action $\alpha$ associated with $b$ :

$$
\left\|\alpha(s) v_{n_{i}}-v_{n_{i}}\right\| \rightarrow 0, \forall s \in S
$$

Proof: First recall [Wolf] that there exists $d>0$ such that $\left|S^{n}\right| \preceq n^{d}$ for all $n$. By an elementary argument5, there exists an infinite sequence $\left(n_{i}\right)$ such that:

$$
\frac{\left|S^{n_{i}+1} \backslash S^{n_{i}}\right|}{\left|S^{n_{i}}\right|} \preceq \frac{1}{\left|n_{i}\right|} .
$$

It follows that the family $\left(S^{n_{i}}\right)_{i}$ is a controlled Følner sequence of $\Gamma$.

Since $\Gamma$ is virtually nilpotent, by Corollary 5.1.3 and Lemma 4.2.2 in [Sha04], it has property $H_{F}$, i.e. every representation with non-zero first reduced cohomology has a finite subrepresentation. Here, by our assumption: $\overline{H^{1}}(\Gamma, \pi)=0$. So the conclusion follows from Corollary 3.7 ,

\footnotetext{
${ }^{4}$ In the conjecture of [Sha04] the assumptions are slightly stronger: $S$ is assumed symmetric, and $\pi$ is supposed to have no finite-dimensional subrepresentation.

${ }^{5}$ If $\left(\left|S^{n+1}\right|-\left|S^{n}\right|\right) /\left|S^{n}\right| \geq d / n$ for all (large) $n$, then $\left|S^{n}\right| \succeq n^{d}$.
} 
Remark: Shalom proved in the final section of [Sha04] that, if the result of Proposition 3.8 was proved under the bare assumption that $\Gamma$ has polynomial growth, this would give rise to a new, simpler 6 proof of Gromov's celebrated theorem Gro1]: a finitely generated group of polynomial growth is virtually nilpotent.

\subsection{A sublinear cocycle with nontrivial reduced 1-cohomo- $\log y$}

It turns out that the converse of Corollary 3.3 is not true in general, for finitely generated groups.

Proposition 3.9. Let $\Gamma$ be a discrete, finitely generated, nonamenable subgroup either in $G=\mathrm{SO}(n, 1)(n \geq 2)$ or $G=\mathrm{SU}(m, 1)(m \geq 1)$. There exists a unitary representation $\sigma$ of $\Gamma$, and $b \in Z^{1}(\Gamma, \sigma)-\overline{B^{1}}(\Gamma, \sigma)$, such that

$$
\|b(g)\| \preceq|g|_{S}^{1 / 2} .
$$

If moreover $\Gamma$ is a cocompact lattice and either $n \geq 3$ or $m \geq 2$, then $\|b(g)\| \sim$ $|g|_{S}^{1 / 2}$ and the representation $\sigma$ may be taken to be irreducible.

Proof: A result of Delorme [Del, Lemme V.5] says that there exists a unitary irreducible representation $\pi$ with $\overline{H^{1}}(G, \pi) \neq 0$ : so we choose $b \in Z^{1}(G, \pi)-$ $\overline{B^{1}}(G, \pi)$. Let $K$ be a maximal compact subgroup of $G$; replacing $b$ by a cohomologous 1-cocycle, we may assume that $\left.b\right|_{K} \equiv 0$. Then $b: G \rightarrow \mathcal{H}_{\pi}$ factors through a map $F: G / K \rightarrow \mathcal{H}_{\pi}$, which is equivariant with respect to the corresponding affine action on $\mathcal{H}_{\pi}$. By an unpublished result of Shalom (for a proof, see Corollary 3.3.10 in [BHV]), the map $F$ is harmonic. We may now appeal to Gromov's results ([Gro2], section 3.7.D'; see also Proposition 3.3.21 in [BHV]) on the growth of harmonic, equivariant maps from a rank 1, Riemannian, symmetric space to a Hilbert space. If $d\left(x, x_{0}\right)$ denotes the Riemannian distance between $x$ and the point $x_{0}$ with stabilizer $K$ in $G / K$, then for some constant $C>0$,

$$
\|F(x)\|^{2}=C d\left(x, x_{0}\right)+o\left(d\left(x, x_{0}\right)\right) .
$$

Set $\sigma=\left.\pi\right|_{\Gamma} ;$ then

$$
\left\|\left.b\right|_{\Gamma}(g)\right\|^{2} \sim d\left(g x_{0}, x_{0}\right) \preceq|g|_{S} .
$$

Let us prove that $\left.b\right|_{\Gamma}$ is not an almost coboundary. First, we appeal to a result of Shalom Sha00, stating that the restriction map $H^{1}(G, \pi) \rightarrow H^{1}(\Gamma, \sigma)$

\footnotetext{
${ }^{6}$ The proof would be simpler in that it would not appeal to the solution of Hilbert's 5th problem about the structure of locally compact groups.
} 
is injective, so that $\left.b\right|_{\Gamma}$ is unbounded. Now $\sigma$, as the restriction to $\Gamma$ of a nontrivial unitary irreducible representation of $G$, does not weakly contain the trivial representation of $\Gamma$ (this follows from a result of Cowling [Cow]: for some $p>1$, all coefficients of $\pi$ are in $L^{p}(G)$. Therefore, a suitable tensor power of $\pi$ is a subrepresentation of $\lambda_{G} \oplus \lambda_{G} \oplus \ldots$, where $\lambda_{G}$ is the regular representation; restricting, a suitable tensor power of $\sigma$ is a sub-representation of $\lambda_{\Gamma} \oplus \lambda_{\Gamma} \oplus \ldots$... Non-amenability of $\Gamma$ allows one to conclude). By Guichardet's well-known criterion (see Guic], Cor. 2.3 in Chap. III), this implies that $B^{1}(\Gamma, \sigma)=\overline{B^{1}(\Gamma, \sigma)}$, in particular every almost coboundary is bounded. Since $\left.b\right|_{\Gamma}$ is unbounded, this gives the desired result.

Finally, if $\Gamma$ is a cocompact lattice in $G$, then $\Gamma$ is quasi-isometric to $G / K$, so we get $\left\|\left.b\right|_{\Gamma}(g)\right\|^{2} \sim|g|_{S}$. Moreover, if $n \geq 3$ or $m \geq 2$, then $\pi$ is not in the discrete series of $G$ (see [Del, Remarque V.8]), so that $\sigma=\left.\pi\right|_{\Gamma}$ is irreducible, by a result of Cowling and Steger [CS, Prop. 2.5].

\subsection{Cocycles with slow growth}

We prove here that, on an a-T-menable group (e.g. Z), there exist cocycles with arbitrarily slow growth.

Proposition 3.10. Assume that $G$ is locally compact, a-T-menable. For every proper function $f: G \rightarrow[1, \infty[$, there exists a continuous conditionally negative definite, proper function $\psi$ on $G$ such that $\psi \leq f$.

This is obtained as a consequence of the following lemma (see $\$ 2.2$ for the definition of Bernstein functions).

Lemma 3.11. Let $u$ be a proper function on $\mathbf{R}_{+}$, with $u(t) \geq 1$ for $t \in \mathbf{R}_{+}$. There exists a proper Bernstein function $F$ such that $F(t) \leq u(t)$ for $t \in \mathbf{R}_{+}$.

Proof: We are going to define inductively a sequence $\left(x_{n}\right)_{n \geq 1}$ of positive real numbers such that $0<x_{n}<2^{-n}$, and define

$$
F(t)=\sum_{n=1}^{\infty}\left(1-e^{-t x_{n}}\right)
$$

Since $1-e^{-t x_{n}} \leq t x_{n}$, the series defining $F$ will converge uniformly on compact subsets of $\mathbf{R}_{+}$, so $F$ will be a Bernstein function (in fact associated with $\mu=$ $\sum_{n=1}^{\infty} \delta_{x_{n}}$ and $\left.a=0\right)$. Let $F_{m}(t)=\sum_{n=1}^{m}\left(1-e^{-t x_{n}}\right)$ be the $m$-th partial sum. For fixed $m$, we will have $F \geq F_{m}$, hence

$$
\liminf _{t \rightarrow \infty} F(t) \geq \lim _{t \rightarrow \infty} F_{m}(t)=m
$$


since this holds for every $m$, we have $\lim _{t \rightarrow \infty} F(t)=\infty$, i.e. $F$ is proper.

It remains to manage to construct the $x_{n}$ 's so that $F \leq u$ on $\mathbf{R}_{+}$. We will construct $x_{n}$ inductively so that $u>F_{n}+2^{-n}$ on $\mathbf{R}_{+}$. Setting $F_{0} \equiv 0$, the construction will also apply to $n=1$. So assume $0<x_{n-1}<2^{-n+1}$ has been constructed so that $u>F_{n-1}+2^{-n+1}$ on $\mathbf{R}_{+}$. Since $u$ is proper and $F_{n-1}$ is bounded, we find $k_{n}>0$ large enough so that $u(t)>F_{n-1}(t)+2$ for $t>k_{n}, t \in$ $\mathbf{R}_{+}$. By taking $x_{n}>0$ very small (with $x_{n}<2^{-n}$ anyway), we may arrange to have $1-e^{-t x_{n}}<2^{-n}$ for $t<k_{n}$. Then, for $t<k_{n}, t \in \mathbf{R}_{+}$:

$$
u(t)-F_{n}(t)=u(t)-F_{n-1}(t)-\left(1-e^{-t x_{n}}\right)>2^{-n+1}-2^{-n}=2^{-n} ;
$$

while for $t \geq k_{n}, t \in \mathbf{R}_{+}$:

$$
u(t)-F_{n}(t)=u(t)-F_{n-1}(t)-\left(1-e^{-t x_{n}}\right)>2-1=1>2^{-n} .
$$

This concludes the induction step.

Proof of Proposition 3.10; If $G$ is compact, we can take $\psi=0$; thus suppose $G$ noncompact. As $G$ is a-T-menable, we may choose a proper conditionally negative definite function $\psi_{0}$ on $G$.

Define a proper function $u \geq 1$ on $\mathbf{R}_{+}$by

$$
u(t)=\inf \left\{f(g): g \in \psi_{0}^{-1}([t, \infty[)\} .\right.
$$

By lemma 3.11, we can find a proper Bernstein function $F$ such that $F \leq u$ on $\mathbf{R}_{+}$. Then, by construction, $F\left(\psi_{0}(g)\right) \leq f(g)$, and by Lemma 2.4, $F \circ \psi_{0}$ is conditionally negative definite.

\subsection{Cocycles with arbitrary large sublinear growth}

As we observed earlier, a cocycle on $\mathbf{Z}^{n}$ (or $\mathbf{R}^{n}$ ) has either linear or sublinear growth. This raises the question whether there is a gap between the two. We show here that it is not the case.

Lemma 3.12. Let $w: \mathbf{R}_{+} \rightarrow \mathbf{R}_{+}$be any function with sublinear growth. Then there exists a sublinear Bernstein function $F$ such that $F(x) \geq w(x)$ for $x$ large enough.

Proof: The function $x \mapsto w(x) / x$ tends to zero. It is easy to construct a decreasing function $x \mapsto u(x)$ of class $C^{1}$, such that $u(x) \geq w(x) / x$ for $x$ large enough, and such that $u(x) \rightarrow 0$ when $x \rightarrow \infty$.

Now define the measure

$$
d \mu(s)=\frac{-u^{\prime}(1 / s)}{s^{3}} 1_{[0,1]}(s) d s .
$$


An immediate calculation gives, for $0<\varepsilon \leq 1, \int_{\varepsilon}^{1} s d \mu(s)=u(1)-u(1 / \varepsilon)$, which is bounded, so that $\int_{0}^{1} s d \mu(s)<\infty$. So we can define the Bernstein function associated to $\mu$ : $F(t)=\int_{0}^{\infty}\left(1-e^{-t s}\right) d \mu(s)$. Then, for all $t \geq 1$, using the inequality $1-e^{-t s} \geq\left(1-e^{-1}\right) t s$ on $[0,1 / t]$ :

$$
\begin{gathered}
F(t) \geq \int_{0}^{1 / t}\left(1-e^{-t s}\right) d \mu(s) \\
\geq\left(1-e^{-1}\right) t \int_{0}^{1 / t} s d \mu(s) \\
=\left(1-e^{-1}\right) t \int_{0}^{1 / t} \frac{-u^{\prime}(1 / s) d s}{s^{2}} \\
=\left(1-e^{-1}\right) t u(t) \\
\geq\left(1-e^{-1}\right) w(t) \quad \text { for large } t .
\end{gathered}
$$

The Bernstein function $x \mapsto\left(1-e^{-1}\right)^{-1} F(x)$ satisfies our purposes, as it is easy to see that it is sublinear.

An example of an application of Lemma 3.12 is the following result.

Proposition 3.13. Let $G$ be a locally compact, compactly generated group having a 1-cocycle of linear growth (e.g. $G=\mathbf{Z}^{n}$ or $\mathbf{R}^{n}$ for $n \geq 1$ ). Then, for every function $f: G \rightarrow \mathbf{R}_{+}$with sublinear growth, there exists on $G$ a sublinear 1 cocycle b such that $\|b(g)\| \succeq f(g)$.

Proof: Let $b^{\prime}$ denote a 1-cocycle with linear growth, and write $|g|=\left\|b^{\prime}(g)\right\|$, so that $g \mapsto|g|$ is equivalent to the word length, and its square is conditionally negative definite on $G$.

By hypothesis, $f(g) \prec|g|$. Define $w: \mathbf{R}_{+} \rightarrow \mathbf{R}_{+}$by

$$
w(x)=\sup \{f(h):|h| \leq x\}
$$

Then $w$ is sublinear on $\mathbf{R}_{+}$, and so is the function $x \mapsto w\left(x^{1 / 2}\right)^{2}$. By Lemma 3.12, we find a sublinear Bernstein function $F$ such that $F(x) \geq w\left(x^{1 / 2}\right)^{2}$ for large $x$. Using Lemma 2.4, the function $g \mapsto F\left(|g|^{2}\right)$ is conditionally negative definite on $G$; moreover $F\left(|g|^{2}\right)^{1 / 2} \prec|g|$, and $F\left(|g|^{2}\right)^{1 / 2} \geq f(g)$ for $g \in G$ with $|g|$ large enough. 


\section{Cocycles with non-slow growth}

\subsection{Amenability}

Here is a generalization of a result by Guentner and Kaminker [GK, §5] who proved it in the case of discrete groups.

Theorem 4.1. Let $G$ be a locally compact group, and $S$ a symmetric, compact generating subset. Suppose that $G$ admits a 1-cocycle $b$ with compression $\rho(r) \succ$ $r^{1 / 2}$. Then $G$ is amenable.

Corollary 4.2. If a locally compact, compactly generated group admits a linear 1-cocycle, then it is amenable.

Proof of Theorem 4.1 For $t>0$, define $f_{t}(g)=e^{-t\|b(g)\|^{2}}$. By Schoenberg's Theorem [BHV, Appendix C], $f_{t}$ is positive definite. We claim that $f_{t}$ is square summable. Denote $S_{n}=\left\{g \in G:|g|_{S}=n\right\}$, and fix a left Haar measure $\mu$ on $G$. There exists $a<\infty$ such that $\mu\left(S_{n}\right) \leq e^{a n}$ for all $n$. Since $\rho(r) \succ r^{1 / 2}$, there exists $n_{0}$ such that, for all $n \geq n_{0}$, and all $g \in S_{n}, 2 t\|b(g)\|^{2} \geq(a+1) n$. Then, for all $n \geq n_{0}$,

$$
\begin{aligned}
& \int_{S_{n}} f_{t}(g)^{2} d \mu(g)=\int_{S_{n}} e^{-2 t\|b(g)\|^{2}} d \mu(g) \\
\leq & \int_{S_{n}} e^{-(a+1) n} d \mu(g) \leq \mu\left(S_{n}\right) e^{-(a+1) n} \leq e^{-n} .
\end{aligned}
$$

Therefore, the sequence $\left(\int_{S_{n}} f_{t}(g)^{2} d \mu(g)\right)$ is summable, so that $f_{t}$ is squaresummable.

By [Dix, Théorème 13.8.6], it follows that there exists a positive definite, square-summable function $\varphi_{t}$ on $G$ such that $f_{t}=\varphi_{t} * \varphi_{t}$, where $*$ denotes convolution. In other words, $f_{t}=\left\langle\lambda(g) \varphi_{t}, \varphi_{t}\right\rangle$, where $\lambda$ denotes the left regular representation of $G$ on $L^{2}(G)$. Note that $f_{t}$ converges to 1 , uniformly on compact subsets, when $t \rightarrow 0$. We conclude that $\left(\varphi_{t}\right)_{t>0}$ provides a net of almost invariant unit vectors for the regular representation of $G$ in $L^{2}(G)$, so that $G$ is amenable.

\subsection{Cocycles with linear growth}

Let us recall a property introduced by Shalom in [Sha04]: a group has Property $H_{F D}$ if every unitary representation such that $\overline{H^{1}}(\Gamma, \pi) \neq 0$ has a finitedimensional subrepresentation.

Here are a few useful results about Property $H_{F D}$. 
1) Property $H_{F D}$ is a quasi-isometry invariant among discrete amenable groups (Shalom, Sha04, Theorem 4.3.3]).

2) A finitely generated amenable group with Property $H_{F D}$ has a finite index subgroup with infinite abelianization [Sha04, Theorem 4.3.1].

3) A connected amenable Lie group has Property $H_{F D}$ (F. Martin, Mar, Theorem 3.3]). A polycyclic group has Property $H_{F D}$ [Sha04, Theorem 5.1.4]. Both results rely on a deep result due to Delorme [Del, Corollaire V.2]: connected solvable Lie groups have Property $H_{F D}$.

4) The semidirect product $\mathbf{Z}[1 / m n] \rtimes_{m / n} \mathbf{Z}$, and the wreath product $F$ ? $\mathbf{Z}$, where $F$ is any finite group, have Property $H_{F D}$ Sha04, Theorems 5.2.1 and 5.3.1]. Semidirect products $\left(\mathbf{R} \oplus \bigoplus_{p \in S} \mathbf{Q}_{p}\right) \rtimes_{\frac{m}{n}} \mathbf{Z}$ or $\left(\bigoplus_{p \in S} \mathbf{Q}_{p}\right) \rtimes_{\frac{m}{n}} \mathbf{Z}$, with $m, n$ co-prime integers, and $S$ a finite set of prime numbers dividing $m n$ also have Property $H_{F D}$ [Sha04, Proof of Theorem 5.3.1].

5) The wreath product $\mathbf{Z} \backslash \mathbf{Z}$ does not have Property $H_{F D}$ Sha04, Theorem $5.4 .1]$.

We prove:

Theorem 4.3. Let $G$ be a locally compact, compactly generated group with Property $H_{F D}$. Suppose that $G$ admits a unitary representation $\pi$ such that $\operatorname{lin}(G, \pi)$ is nonempty. Then, $G$ has a compact normal subgroup $K$ such that $G / K$ is isomorphic to some closed subgroup of $\operatorname{Isom}\left(\mathbf{R}^{n}\right)$. In particular,

- $G$ is quasi-isometric to $\mathbf{R}^{m}$ for some unique $m$,

- If $G$ is discrete, then $G$ is virtually abelian.

Proof: Let $(\mathcal{H}, \pi)$ be a unitary representation of $G$ and suppose that there exists $b \in \operatorname{lin}(G, \pi)$. Replacing $G$ by $G / K$ for some compact normal subgroup if necessary, we can suppose by [Com, Theorem 3.7] that $G$ is separable, and thus we can also assume that $\mathcal{H}$ is separable.

As $G$ has Property $H_{F D}, \mathcal{H}$ splits into a direct sum $\mathcal{H}=\mathcal{H}^{\prime} \oplus\left(\bigoplus_{n \in \mathbf{N}} \mathcal{H}_{n}\right)$ where $\mathcal{H}_{n}$ are finite dimensional subrepresentations and where $\mathcal{H}^{\prime}$ is a subrepresentation with trivial reduced cohomology. By Proposition 3.1, and since $b$ has linear growth, its orthogonal projection on $\oplus_{n \in \mathbf{N}} \mathcal{H}_{n}$ still has linear growth, so we can assume that $\mathcal{H}=\oplus_{n \in \mathbf{N}} \mathcal{H}_{n}$. Now, let $b_{n}$ be the projection of $b$ on $\oplus_{k \leq n} \mathcal{H}_{k}$. Then $b_{n} \rightarrow b$ uniformly on compact subsets. So, as $\operatorname{lin}(G, \pi)$ is open 
and $b \in \operatorname{lin}(G, \pi)$, there exists $n$ such that $b_{n} \in \operatorname{lin}(G, \pi)$. Hence $b_{n}$ defines a proper morphism $G \rightarrow \operatorname{Isom}\left(\mathcal{H}_{n}\right)$; denote by $H$ its image.

If $G$ is discrete, by Bieberbach's Theorem (see for instance [Bus]), this implies that $G$ has a homomorphism with finite kernel onto a virtually abelian group, hence is itself virtually abelian.

In general, by Corollary B.2, $G$ acts properly and cocompactly on some Euclidean space $\mathbf{R}^{n}$, hence is quasi-isometric to $\mathbf{R}^{n}$.

\subsection{Uniform embeddings into Hilbert spaces}

Let $G$ be a locally compact group, and $|\cdot|_{S}$ the length function with respect to a compact symmetric generating subset $S$. For an arbitrary map $f$ of $G$ to a Hilbert space $\mathcal{H}$, define its dilation as:

$$
\delta(x)=\sup \left\{\|f(g)-f(h)\|:\left|g^{-1} h\right|_{S} \leq x\right\} \in \mathbf{R}_{+} \cup\{\infty\},
$$

and its compression as:

$$
\rho(x)=\inf \left\{\|f(g)-f(h)\|:\left|g^{-1} h\right|_{S} \geq x\right\} \in \mathbf{R}_{+} \cup\{\infty\},
$$

We call $f$ a uniform map if $\delta(x)<\infty$ for all $x \in \mathbf{R}_{+}$(by an easy standard argument, this implies that $\delta$ has at most linear growth). The map $f$ is called a uniform embedding if, in addition, $\rho(x) \rightarrow \infty$ when $x \rightarrow \infty$. It is called a quasi-isometric embedding if, in addition, it has compression with linear growth, i.e. $\rho(r) \succeq r$.

The following result, which was pointed out to us by M. Gromov (who provided a proof in the discrete case), is very useful.

Proposition 4.4. Let $G$ be a locally compact, compactly generated, amenable group. Let $f$ be a uniform map of $G$ into a Hilbert space, and $\rho$ its compression, $\delta$ its dilation. Then $G$ admits a 1-cocycle with compression $\geq \rho-a$ and dilation $\leq \delta+a$, for some constant $a \geq 0$. If $G$ is discrete, we can take $a=0$.

Proof: Let $m$ be a mean on $G$, that is, a continuous, linear map on $L^{\infty}(G)$ such that $m(1)=1, m(f) \geq 0$ whenever $f \geq 0$ locally almost everywhere. Since $G$ is amenable, we choose $m$ to be right invariant, i.e. $m(f \cdot g)=m(f)$ for all $g \in G$ and $f \in L^{\infty}(G)$, where $(f \cdot g)(h)$ is by definition equal to $f\left(h g^{-1}\right)$.

For $g, h \in G$, set $\Psi(g, h)=\|f(g)-f(h)\|^{2}$. By assumption,

$$
\rho\left(\left|g^{-1} h\right|_{S}\right) \leq \Psi(g, h)^{1 / 2} \leq \delta\left(\left|g^{-1} h\right|_{S}\right), \quad \forall g, h \in G
$$


By Lemma A.1 in the appendix, there exists a uniformly continuous function $f^{\prime}$ at bounded distance from $f$ (if $G$ is discrete we do not need Lemma A.1 since it suffices to take $\left.f^{\prime}=f\right)$. Write $\Psi^{\prime}(g, h)=\left\|f^{\prime}(g)-f^{\prime}(h)\right\|^{2}$. Then $\Psi^{1 / 2}-\left(\Psi^{\prime}\right)^{1 / 2}$ is bounded.

Now set $u_{g_{1}, g_{2}}(h)=\Psi^{\prime}\left(h g_{1}, h g_{2}\right)$ for $g_{1}, g_{2}, h \in G$. The upper bound by $\delta$ and the uniform continuity of $f^{\prime}$ imply that the mapping $\left(g_{1}, g_{2}\right) \mapsto u_{g_{1}, g_{2}}$ is a continuous function from $G \times G$ to $L^{\infty}(G)$, so that the function $\Psi_{m}\left(g_{1}, g_{2}\right)=$ $m\left(u_{g_{1}, g_{2}}\right)$ is continuous on $G \times G$.

For $g_{1}, \ldots, g_{n} \in G$ and $\lambda_{1}, \ldots, \lambda_{n} \in \mathbf{R}$ with $\sum_{i=1}^{n} \lambda_{i}=0$, we have

$$
\sum_{i, j} \lambda_{i} \lambda_{j} \Psi_{m}\left(g_{i}, g_{j}\right)=\sum_{i, j} \lambda_{i} \lambda_{j} m\left(u_{g_{i}, g_{j}}\right)=m\left(\sum_{i, j} \lambda_{i} \lambda_{j} u_{g_{i}, g_{j}}\right) \leq 0
$$

because $m$ is positive and $\sum_{i, j} \lambda_{i} \lambda_{j} u_{g_{i}, g_{j}}$ is a non-positive function on $G$ (since $\Psi^{\prime}$ is a conditionally negative definite kernel). So $\Psi_{m}$ is a conditionally negative definite kernel.

Since $m$ is right $G$-invariant, it follows that $\Psi_{m}$ is $G$-invariant, so that we can write $\Psi_{m}\left(g_{1}, g_{2}\right)=\psi\left(g_{1}^{-1} g_{2}\right)$ for some continuous, conditionally negative definite function $\psi$ on $G$. Let $b$ be the corresponding 1-cocycle. The estimates on $\psi$, and thus on $\|b\|$, follow from the positivity of $m$.

Corollary 4.5. If a locally compact, compactly generated amenable group $G$ quasi-isometrically embeds into a Hilbert space, then it admits a 1-cocycle with linear growth.

From Corollary 4.5] and Theorem 4.3, we deduce immediately:

Corollary 4.6. Let $G$ be a locally compact, compactly generated amenable group with property $H_{F D}$. The group $G$ admits a quasi-isometric embedding into a Hilbert space if and only if $G$ acts properly on a Euclidean space. In particular, if $G$ is discrete, this means that it is virtually abelian.

Combining this corollary with Shalom's results mentioned in $\$ 4.2$, we immediately obtain Theorem 1.4 in the introduction.

Proof of Corollary 1.5. We must prove that if a finitely generated group $\Gamma$ is quasi-isomorphic to $\mathbf{Z}^{n}$, then it has a finite index subgroup isomorphic to $\mathbf{Z}^{n}$. This result is known as a consequence of Gromov's polynomial growth Theorem (see e.g. [GH, Théorème 1.17], with a sketch of proof on p. 13); it has been given a new proof in [Sha04]. As in [Sha04], the first step is the fact that, since Property $H_{F D}$ is a quasi-isometric invariant of amenable groups, $\Gamma$ has Property $H_{F D}$. Now, being quasi-isometric to $\mathbf{Z}^{n}, \Gamma$ quasi-isometrically embeds into a Hilbert space, hence is virtually isomorphic to $\mathbf{Z}^{m}$ for some $m$, by Theorem 1.4 . 
Finally, it is well-known that $\mathbf{Z}^{m}$ and $\mathbf{Z}^{n}$ being quasi-isometric implies $m=n$. For instance, it suffices to observe that the degree of growth of $\mathbf{Z}^{n}$ is $n$.

Proof of Corollary 1.6. It is enough to show that the regular tree of degree 3 does not embed quasi-isometrically into a Hilbert space. But such a tree is quasi-isometric to $G=\mathrm{Q}_{2} \rtimes_{2} \mathbf{Z}$, since this group acts cocompactly and properly on the Bass-Serre tree of $\mathrm{SL}_{2}\left(\mathbf{Q}_{2}\right)$. On the other hand, $\mathbf{Q}_{2} \rtimes_{2} \mathbf{Z}$ has no non-trivial compact normal subgroup (indeed, such a subgroup would be contained in $\mathbf{Q}_{2}$, in which the non-trivial conjugacy classes of $G$ are unbounded), so it does not act properly cocompactly on a Euclidean space. Since it has Property $H_{F D}$, by Theorem 1.4, it does not quasi-isometrically embed into a Hilbert space.

Remark 4.7. Proposition 4.4 is specific to amenable groups. For instance, if $\Gamma$ is a free group on $n \geq 2$ generators, then it has uniform embeddings with compression $\geq|g|^{a}$ for arbitrary $a<1$ [GK, §6], while it has no 1-cocycle with compression $\succ|g|^{1 / 2}$ since it is non-amenable ([GK, §5] or Theorem 4.1).

\section{A Maps into Hilbert spaces}

Let $G$ be a locally compact group and $\mathcal{H}$ a Hilbert space. Let $f$ be a map: $G \rightarrow \mathcal{H}$ (not necessarily continuous). We call $f$ a uniform map if, for every compact subset $K \subset G$, we have $\sup _{g \in G, k \in K}\|f(k g)-f(g)\|<\infty$. If $G$ is compactly generated, this coincides with the definition given in $\$ 4.3$.

Lemma A.1. Let $f: G \rightarrow \mathcal{H}$ be a uniform map. Then there exists $\tilde{f}: G \rightarrow \mathcal{H}$ such that:

- $\tilde{f}$ is at bounded distance from $f$, and

- $\tilde{f}$ is uniformly continuous on $G$.

Proof: Fix an open, relatively compact, symmetric neighbourhood $V$ of 1 in $G$. Consider a closed, discrete subset $X \subset G$ such that

(1) $\bigcup_{x \in X} x V=G$, and

(2) for all $x, y \in X$, if $x^{-1} y \in V$, then $x=y$.

The existence of such a subset $X$ is immediate from Zorn's Lemma.

\footnotetext{
${ }^{7}$ It follows from the proof that we can take any Banach space instead of $\mathcal{H}$.
} 
Fix a function $\phi: G \rightarrow \mathbf{R}_{+}$, continuous with compact support, such that $\phi \leq 1$, and (3): $\phi \equiv 1$ on $V$. Fix a symmetric, compact subset $W$ containing the support of $\phi$.

Set $\Phi(g)=\sum_{x \in X} \phi\left(x^{-1} g\right)$ and observe that $\Phi(g) \geq 1$ as a consequence of (1) and (3). Define

$$
\tilde{f}(g)=\frac{1}{\Phi(g)} \sum_{x \in X} \phi\left(x^{-1} g\right) f(x) .
$$

Let us first check that $\tilde{f}$ is at bounded distance from $f$. For all $g \in G$,

$$
\tilde{f}(g)-f(g)=\sum_{x \in X} \frac{\phi\left(x^{-1} g\right)}{\Phi(g)}(f(x)-f(g)) .
$$

Since $f$ is a uniform map, there exists $M<\infty$ such that for all $g, h \in G$, $h^{-1} g \in W$ implies $\|f(h)-f(g)\| \leq M$. It follows that, for all $g \in G$, we have $\|\tilde{f}(g)-f(g)\| \leq M$.

Now let us show that $f$ is uniformly continuous. Consider a neighbourhood $V_{0}$ of 1 in $G$ such that $V_{0}^{2} \subset V$. It immediately follows that, for every $g \in G$, the set $X \cap g V_{0}$ contains at most one element. On the other hand, by compactness, there exist $g_{1}, \ldots, g_{n}$ such that $W \subset \bigcup_{i=1}^{n} g_{i} V_{0}$. It follows that, for all $g \in G$, the set $g W \cap X$ has cardinality at most $n$.

Write $u_{\phi}(g)=\sup _{h \in G}|\phi(h)-\phi(h g)|$. Since $\phi$ is uniformly continuous, $u_{\phi}(g) \rightarrow$ 0 when $g \rightarrow 1$.

Then $\left|\phi\left(x^{-1} g\right)-\phi\left(x^{-1} h\right)\right| \leq u_{\phi}\left(g^{-1} h\right)$ and, for all $g, h \in G, \Phi(g)-\Phi(h)=$ $\sum_{x \in X}\left(\phi\left(x^{-1} g\right)-\phi\left(x^{-1} h\right)\right) \leq 2 n u_{\phi}\left(g^{-1} h\right)$, since the only nonzero terms are those for $x \in(g W \cap X) \cup(h W \cap X)$. Accordingly, $\Phi$ is uniformly continuous. Since $\Phi \geq 1$, it follows that $1 / \Phi$ is also uniformly continuous; let us define $u_{1 / \Phi}$ as we have defined $u_{\phi}$.

For $g, h \in G$,

$$
\begin{gathered}
\left|\frac{\phi\left(x^{-1} g\right)}{\Phi(g)}-\frac{\phi\left(x^{-1} h\right)}{\Phi(h)}\right| \leq\left|\frac{\phi\left(x^{-1} g\right)}{\Phi(g)}-\frac{\phi\left(x^{-1} h\right)}{\Phi(g)}\right|+\left|\frac{\phi\left(x^{-1} h\right)}{\Phi(g)}-\frac{\phi\left(x^{-1} h\right)}{\Phi(h)}\right| \\
\leq \frac{\left|\phi\left(x^{-1} g\right)-\phi\left(x^{-1} h\right)\right|}{\Phi(g)}+\left|\phi\left(x^{-1} h\right)\right|\left|\frac{1}{\Phi(g)}-\frac{1}{\Phi(h)}\right| \\
\leq u_{\phi}\left(g^{-1} h\right)+u_{1 / \Phi}\left(g^{-1} h\right) .
\end{gathered}
$$

Therefore, fixing some $x_{0} \in X$,

$$
\|\tilde{f}(g)-\tilde{f}(h)\|=\sum_{x \in X}\left(\frac{\phi\left(x^{-1} g\right)}{\Phi(g)}-\frac{\phi\left(x^{-1} h\right)}{\Phi(h)}\right)\left(f(x)-f\left(x_{0}\right)\right)
$$




$$
\begin{gathered}
\leq \sum_{x \in X}\left|\frac{\phi\left(x^{-1} g\right)}{\Phi(g)}-\frac{\phi\left(x^{-1} h\right)}{\Phi(h)}\right|\left\|f(x)-f\left(x_{0}\right)\right\| \\
\leq \sum_{x \in(g W \cap X) \cup(h W \cap X)}\left|\frac{\phi\left(x^{-1} g\right)}{\Phi(g)}-\frac{\phi\left(x^{-1} h\right)}{\Phi(h)}\right|\left\|f(x)-f\left(x_{0}\right)\right\| \\
\leq\left(u_{\phi}\left(g^{-1} h\right)+u_{1 / \Phi}\left(g^{-1} h\right)\right) \sum_{x \in(g W \cap X) \cup(h W \cap X)}\left\|f(x)-f\left(x_{0}\right)\right\| .
\end{gathered}
$$

Since $f$ is a uniform map, there exists $M^{\prime}<\infty$ such that $h^{-1} g \in V^{2} W$ implies $\|f(h)-f(g)\| \leq M^{\prime}$ for all $g, h \in G$. Now fix $x_{0}$ so that $g \in x_{0} V$, and suppose $g^{-1} h \in V$. If $x \in g W \cup h W$, then it follows that $\left\|f(x)-f\left(x_{0}\right)\right\| \leq M^{\prime}$. Accordingly, whenever $g^{-1} h \in V$,

$$
\|\tilde{f}(g)-\tilde{f}(h)\| \leq 2 n\left(u_{\phi}\left(g^{-1} h\right)+u_{1 / \Phi}\left(g^{-1} h\right)\right) M^{\prime},
$$

so that $\tilde{f}$ is uniformly continuous.

\section{B Actions on Euclidean spaces}

Proposition B.1. Let $G$ be a closed subgroup of $\mathrm{E}_{n}(\mathbf{R})=\operatorname{Isom}\left(\mathbf{R}^{n}\right)$. The following are equivalent:

(i) $G$ is cocompact in $\mathrm{E}_{n}(\mathbf{R})$.

(ii) $G$ acts cocompactly on $\mathbf{R}^{n}$.

(iii) $G$ does not preserve any proper affine subspace of $\mathbf{R}^{n}$.

Proof: (i) $\Leftrightarrow($ ii $) \Rightarrow$ (iii) are trivial.

Let us show (iii) $\Rightarrow$ (ii). We use some results of Guivarc'h on the structure of closed (not necessarily connected) subgroups of amenable connected Lie groups. By [Guiv, Théorème IV.3 and Lemma IV.1], $G$ has a characteristic closed cocompact solvable subgroup $R$. Then $R$ has a characteristic subgroup of finite index $N$ which maps to a torus of $\mathrm{O}_{n}(\mathbf{R})$ through the natural projection $\mathrm{E}_{n}(\mathbf{R}) \rightarrow \mathrm{O}_{n}(\mathbf{R})$.

First case: $G$ does not contain any nontrivial translation. Then $N$ is abelian. If $g \in N$, let $d_{g}$ denote its displacement length: $d_{g}=\inf \left\{\|g \cdot v-v\|: v \in \mathbf{R}^{n}\right\}$. The subset $A_{g}=\left\{v \in \mathbf{R}^{n}:\|g v-v\|=d_{g}\right\}$ is a (nonempty) affine subspace of $\mathbf{R}^{n}$, and is $N$-stable since $N$ is abelian. Also note that if $W$ is any $g$-stable 
affine subspace, then $8 \cap A_{g} \neq \emptyset$. It easily follows that finite intersections of subspaces of the form $A_{g}$, for $g \in N$, are nonempty, and a dimension argument immediately yields that $A=\bigcap_{g \in N} A_{g} \neq \emptyset$. This is a $G$-invariant affine subspace, hence is, by assumption, all of $\mathbf{R}^{n}$. Therefore, every element in $N$ is a translation, so that $N=\{1\}$ and thus $G$ is compact. This implies that $G$ has a fixed point, so that the assumption implies $n=0$ (i.e. leads to a contradiction if $n \geq 1$ ).

General case. Argue by induction on the dimension $n$. Suppose that $n \geq 1$. Let $T_{G}$ be the subgroup of translations in $G$. Let $W$ be the linear subspace generated by $T_{G}$. Since $T_{G}$ is closed, it acts cocompactly on $W$. Moreover, by the first case, $W$ has positive dimension. Note that the linear action of $G$ clearly preserves $W$.

Now look at the action of $G$ on the affine space $\mathbf{R}^{n} / W$. It does not preserve any proper affine subspace, hence is cocompact by the induction hypothesis. Since the action of $T_{G}$ on $W$ is also cocompact, it follows that the action of $G$ on $\mathbf{R}^{n}$ is also cocompact.

Corollary B.2. Let $G$ be a locally compact group. Suppose that $G$ has a proper isometric action on a Euclidean space. Then it has a proper cocompact isometric action on a Euclidean space.

Proof: Let $G$ act on a Euclidean space by isometries. Let $V$ be a $G$-invariant affine subspace of minimal dimension. Then the action of $G$ on $V$ is clearly proper, and is cocompact by Proposition B.1.

\section{References}

[BHV] M. Bachir BekKa, Pierre de la Harpe, Alain Valette. "Kazhdan's Property (T)". Book to appear at Cambridge Univ. Press, currently available at http://poncelet.sciences.univ-metz.fr/ bekka/, 2004.

[BeSc] I. Benjamini and O. Schramm. (1997). Every graph with a positive Cheeger constant contains a tree with a positive Cheeger constant. Geom. Funct. Ann. 7, 403-419.

\footnotetext{
${ }^{8}$ Let $v \in A_{g}$ such that $d(v, W)=d\left(A_{g}, W\right)$. Let $p$ denote the projection on $W$; since $W$ is $g$ stable, $p$ commutes with $g$. Since $d(v, p v)=d(g v, g p v) \leq d(x, y)$ for all $x \in[v, g v], y \in[p v, g p v]$, we easily obtain that $v, g v, p v, g p v$ form a rectangle, so that $d(p v, g p v)=d(v, g v)$ and thus $p v \in A_{g}$ by definition of $A_{g}$.
} 
[BF] Christian Berg, Gunnar Forst. "Potential Theory on Locally Compact Abelian Groups". Springer-Verlag, 1975.

[Bus] Peter Buser. A geometric proof of Bieberbach's theorems on crystallographic groups. L'Enseignement Math. 31, 137-145, 1985.

[Bou] Jean Bourgain. The metrical interpretation of superreflexivity in $B a-$ nach spaces. Israel J. Math. 56(2), 222-230, 1986.

[CCJJV] Pierre-Alain Cherix, Michael Cowling, Paul Jolissaint, Pierre Julg, Alain VAlette. "Groups with the Haagerup Property". Birkhäuser, Progress in Mathematics 197, 2001.

[Com] W. Wistar Comfort. Topological groups. p.1143-1263 in: "Handbook of Set-Theoretic Topology", edited by K. Kunen and J. E. Vaughan, North Holland, Amsterdam, 1984.

[Cow] Michael Cowling. Sur les coefficients des représentations unitaires des groupes de Lie simples, Springer Lect. Notes in Math., 739, 132-178, 1979.

[CS] Michael Cowling, T. STEGER. The irreducibility of restrictions of unitary representations to lattices, J. reine angew. Math., 420, 85-98, 1991.

[Del] Patrick Delorme. 1-cohomologie des représentations unitaires des groupes de Lie semi-simples et résolubles. Produits tensoriels continus de représentations. Bull. Soc. Math. France 105, 281-336, 1977.

[Dix] Jacques Dixmier. Les $C^{*}$-algèbres et leurs représentations. GauthierVillars, Paris, 1969.

[Far] Benson FARB. The extrinsic geometry of subgroups and the generalized word problem. Proc. London Math. Soc. 68(3), 577-593, 1994.

[GH] Etienne GHYs, Pierre DE LA HARPe, eds. "Sur les groupes hyperboliques d'après Mikhael Gromov". Birkhäuser, Progress in Mathematics 83, 1990.

[Gro1] Misha Gromov. Groups of polynomial growth and expanding maps. Publ. Math. IHES, 53, 53-73, 1981.

[Gro2] Misha Gromov. Random Walk in Random Groups. Geom. Funct. Anal. 13(1), 73-146, 2003. 
[GK] Erik Guentner, Jerome KAminker. Exactness and uniform embeddability of discrete groups. J. London Math. Soc. 70, 703-718, 2004.

[Guic] Alain Guichardet. "Cohomologie des groupes topologiques et des algèbres de Lie". Paris, Cédic-Nathan, 1980.

[Guiv] Yves GuIvarc'H. Croissance polynomiale et périodes des fonctions harmoniques. Bull. Soc. Math. France 101, 333-379, 1973.

[HV] Pierre DE la Harpe, Alain Valette. La propriété (T) de Kazhdan pour les groupes localement compacts, Astérisque 175, SMF, 1989.

[Mar] Florian MARTIN. Reduced 1-cohomology of connected locally compact groups and applications. J. Lie Theory, 16, 311-328, 2006.

[Pau] Scott D. Pauls. The large scale geometry in nilpotent Lie groups. Commun. Anal. Geom. 9(5), 951-982, 2001.

[Sch] Isaac J. SchoenberG. Metric spaces and completely monotone functions. Annals of Math. 39(4), 811-841, 1938.

[Sha00] Yehuda SHALOM. Rigidity, unitary representations of semisimple groups, and fundamental groups of manifolds with rank one transformation group. Annals of Math. 152, 113-182, 2000.

[Sha04] Yehuda Shalom. Harmonic analysis, cohomology, and the large scale geometry of amenable groups. Acta Mathematica 193, 119-185, 2004.

[Tes1] Romain TesserA. Asymptotic isoperimetry on groups and uniform embeddings into Banach spaces. Preprint, 2006.

[Tes2] R. Tessera. Large-scale Sobolev inequalities on metric measure spaces. Preprint, 2006.

[Wolf] Joseph A. Wolf. Growth of finitely generated solvable groups and curvature of Riemanniann manifolds. J. Differential Geometry 2 421446, 1968.

Yves de Cornulier

IRMAR, Campus de Beaulieu,

F-35042 Rennes Cedex, France

E-mail: decornul@clipper.ens.fr 
Romain Tessera

Department of mathematics, Vanderbilt University,

Stevenson Center, Nashville, TN 37240, USA,

E-mail: tessera@clipper.ens.fr

Alain Valette

Institut de Mathématiques - Université de Neuchâtel

Rue Emile Argand 11, CH-2007 Neuchâtel, Switzerland

E-mail: alain.valette@unine.ch 